ESJ Natural/Life/Medical Sciences

\title{
Accès À La Terre Et Profil De La Pauvreté Dans Le Delta Du Fleuve Sénégal
}

\author{
El Hadji Malick Sylla \\ Université Gaston Berger de Saint-Louis \\ Karalan Sy \\ Université du Sine Saloum El Hadj Ibrahima Niass \\ Sidy Mohamed Seck \\ Université Gaston Berger de Saint-Louis
}

Doi:10.19044/esj.2021.v17n3p360

Submitted: 23 November 2020

Accepted: 21 January 2021

Published: 31 January 2021
Copyright 2021 Author(s)

Under Creative Commons BY-NC-ND

4.0 OPEN ACCESS

Cite As:

El Hadji M.S., Sidy M.S. \& Karalan S.(2021). Accès À La Terre Et Profil De La Pauvreté Dans Le Delta Du Fleuve Sénégal. European Scientific Journal, ESJ, 17(3), 360.

https://doi.org/10.19044/esj.2021.v17n3p360

\section{Résumé}

Cet article tente de caractériser les ménages agricoles pauvres dans le Delta du fleuve Sénégal après la crise alimentaire de 2008, durant laquelle les politiques publiques ont réalisé des aménagements hydroagricoles pour lutter contre la pauvreté et l'insécurité alimentaire. Il s’appuie sur des enquêtes de terrain auprès des producteurs. Les résultats montrent une forte inégalité entre les cultivateurs en termes de capital foncier et de surfaces cultivées. Aussi les ménages les plus pauvres sont-ils ceux qui n'emblavent pas assez de superficies, qui ne cultivent qu'en contre-saison chaude et, par conséquent, ne gagnent pas assez de revenus agricoles pour améliorer leur condition de vie.

Mots Clés : Pauvreté, Foncier Irrigué, Delta Du Fleuve Sénégal, Partenariat Public-Privé 


\title{
Land Access And Poverty Profile In The Senegal River Delta
}

\author{
El Hadji Malick Sylla \\ Université Gaston Berger de Saint-Louis \\ Karalan Sy \\ Université du Sine Saloum El Hadj Ibrahima Niass \\ Sidy Mohamed Seck \\ Université Gaston Berger de Saint-Louis
}

\begin{abstract}
This paper attempts to characterise poor farming households in the Senegal River Delta after the 2008 food crisis, during which public policies implemented hydro-agricultural developments to combat poverty and food insecurity. It is based on field surveys of producers. The results show a strong inequality between farmers in terms of land capital and cultivated areas. As a result, the poorest households are those that do not cultivate enough land, only cultivate in the off-season and therefore do not earn enough agricultural income to improve their living conditions.
\end{abstract}

Keyword: Poverty, Irrigated Land, Senegal River Delta, Public-Private Partnership

\section{Introduction}

L'accès au foncier pour toutes les catégories sociales a été au cœur de la mise en valeur du Delta comme tout le reste de la Vallée du fleuve Sénégal. L'option des politiques publiques de développer l'irrigation dans les années 1970 et 1980 a permis de passer d'un système agricole traditionnel de décrue à une agriculture irriguée avec une maîtrise complète de l'eau. La gestion des périmètres a modifié l'organisation sociale de la production. Elle a surtout permis de réduire les inégalités sociales d'accès à la terre dans la région. En effet, l'attribution des parcelles a remis en question des droits fonciers coutumiers (droit de maître des terres, et droits de culture) exercés par certains clans nobles au détriment des familles de dépendants (captifs, descendants de captifs, affranchis, clients, etc.). Ainsi, "les différentes catégories sociales... se sont ainsi retrouvées pour cultiver les périmètres irrigués côte à côte sur un pied d'inégalité, avec les mêmes droits et les mêmes obligations » (Boutillier, 1989 : 477).

Dans les années 1990 et au début des années 2000, l'accès à la terre ne sera plus conditionné par la classe sociale mais plutôt par la situation économique des producteurs. La libéralisation des années 1990 a entrainé l'émergence des Périmètres Irrigués Privés (PIP) qui vont permettre aux 
producteurs les plus riches et/ou les plus influents de disposer d'importantes superficies (Bélières et Touré, 1999; Seck, 2009). Le Roy (2006) a étudié les inégalités entre agriculteurs familiaux en mettant en corrélation superficie cultivée et revenu. Il constate que les ménages possédant de faibles surfaces agricoles ne gagnent pas assez pour s'affranchir de la pauvreté alors que les revenus agricoles se concentrent entre les mains de quelques agriculteurs qui détiennent les plus grandes surfaces cultivables. Parallèlement au développement des PIP, l'Etat va encourager l'implantation d'agro-industries (Sy, 2014) dans le Delta du Sénégal. Ces entreprises d'agrobusiness qui produisent sur de vastes périmètres vont participer à la réduction de l'accès au foncier irrigué aux exploitants familiaux les plus démunis (Bourgoin et al., 2016 ; Soullier et al., 2019).

Après la crise alimentaire de 2008, des projets bâtis autour du Partenariat Public-Privé (PPP) ont réalisé de nouveaux aménagements hydroagricoles dans le Delta du Sénégal. Il s'agit notamment du Programme de Développement des Marchés Agricoles du Sénégal (PDMAS) et du Projet de Promotion du Partenariat Rizicole dans le Delta (3PRD) (SAED, 2009, Drieu et al., 2011). Chaque projet a aménagé 2500 ha aux profits de différentes catégories de producteurs choisis après un processus de sélection rigoureux. L’objectif de cet article est de dégager un profil des ménages agricoles pauvres dans le Delta du Sénégal en analysant et discutant les relations entre la pauvreté, l'accès à la terre et les revenus agricoles quelques années après la fin de ces deux projets. L’hypothèse qui sous-tend cette étude est que malgré le Partenariat Public-Privé, la majorité des ménages continue de produire sur de petites superficies et ne parvient toujours pas à gagner assez de revenus agricoles pour améliorer leur niveau de vie.

\section{Matériels Et Méthodes}

L'étude a été menée dans le Delta-central et le Bas Delta correspondant aux communes de Diama et de Ross Béthio. Le choix de ces communes s'explique par le fait qu'elles ont bénéficié de toutes les générations d'aménagements hydroagricoles réalisés dans le Delta. La sélection des localités enquêtées répond à plusieurs critères dont la position géographique par rapport aux terroirs et aux superficies irriguées, l'ethnie de la population et son poids démographique (figure 1). Au total, 326 ménages répartis dans 20 localités ont été enquêtés entre août et septembre 2019. Dans chaque village, un tirage aléatoire simple a été fait sur un échantillon stratifié et tenant compte du nombre de ménages. Cette enquête a ciblé les exploitations 
familiales ${ }^{1}$ (EXFAM) qui pratiquent l'agriculture irriguée dans le Delta du Sénégal.

Figure 1. Situation des localités enquêtées dans le delta du fleuve Sénégal

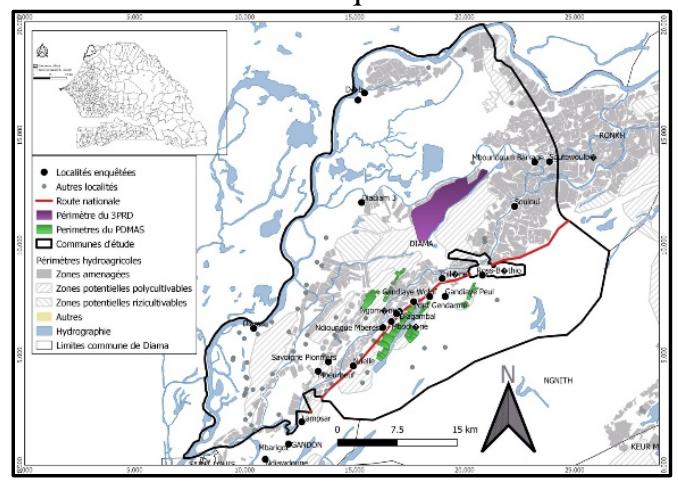

Source: auteurs

Les données recueillies ont été traitées avec le logiciel d'analyse statistique Statistical Package for the Social Sciences (SPSS). Cela a permis de faire une description des parcelles recensées. Pour approfondir notre étude sur l'accès au foncier, nous avons cherché à comprendre deux aspects de la propriété foncière : le canal d'accès à chaque parcelle et la distribution des catégories dimensionnelles des parcelles dans l'échantillon étudié. Pour faciliter l'analyse trois classes ont été retenues : la classe «petite parcelle » regroupe les champs de moins de $5 \mathrm{ha}$; la classe «moyenne parcelle»: concentre les exploitations comprises entre 5 et 20 ha; la classe «grande parcelle » contient les unités de production agricole supérieure à 20 ha. Afin d'établir un profil des ménages pauvres dans la zone étudiée à partir de leur patrimoine foncier, de leur niveau de vie et leur activité agricole, nous avons eu recours à une Classification Hiérarchique Ascendante (CAH). Cette dernière est une méthode statistique qui permet de regrouper en différentes classes les ménages présentant une certaine similitude. Ainsi, sept variables quantitatives ont été sélectionnées pour faire cette analyse typologique : «le nombre des membres du ménage », "la marge (bénéfice) réalisée toutes les trois saisons (FCFA)», «la marge réalisée durant la contre-saison chaude (FCFA) », « la superficie cultivée lors des trois saisons (ha)», « la superficie cultivée lors de la contre-saison chaude (ha)», «les dépenses moyennes par mois (FCFA) des ménages» et «le taux de pauvreté» calculé à partir de l'indice de probabilité de pauvreté (PPI) après l'administration du questionnaire aux ménages (voir IPA, 2018). La méthode de Ward a été

${ }^{1}$ En fait, la loi d'orientation agro-sylvo-pastorale (LOASP) ne distingue que deux types d'exploitation agricole au Sénégal : l'exploitation agricole familiale qui représente environ $90 \%$ des formes d'exploitation et l'agro-business/agriculture industrielle qui peut appartenir à des investisseurs nationaux comme internationaux (République du Sénégal, 2004 ; 2014). 
choisie comme technique d'agrégation et le carré de la distance Euclidienne comme intervalle. Les données ont été par la suite transformées en valeur à travers la standardisation par valeurs centrées réduites.

\section{Résultats}

\section{De la MAS à l'avènement du Partenariat Public-Privé ; un front irrigué en progression}

La carte ci-dessous résume l'évolution des superficies aménagées dans notre zone d'étude depuis 1965 à nos jours (figure 2).

Figure 2. Progression et répartition des aménagements hydroagricoles dans l'espace

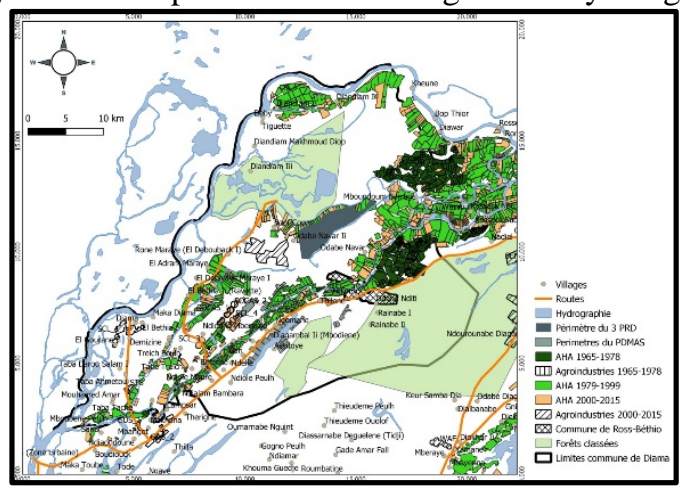

Source : auteurs

Hormis les périmètres occupés par les agro-industries, on peut relever quatre générations d'aménagements hydroagricoles réalisés progressivement. La Mission d’Aménagement du Sénégal (MAS) a mis en place les premiers grands aménagements hydroagricoles dans les années 1950, avec notamment un casier de 6400 ha à Richard-Toll (Bélières et Touré, 1999 ; Seck, 2009). Mais c'est surtout après les indépendances que le front irrigué a pris plus d'ampleur dans le Delta du fleuve Sénégal.

Les années 1960-1970 sont marquées par la création de plusieurs aménagements en submersion contrôlée à Savoigne, Boundoum, Kassack Sud, Tiagar et Grande Digue Tellel. À partir de 1972 ces derniers ont progressivement été transformés en aménagement avec une maîtrise complète de l'eau. Les aménagements de 1979-1999 constituent un tournant dans la progression du front irrigué. En effet, une nouvelle génération d'aménagements hydroagricoles va être mise en place dans le Delta du fleuve Sénégal à partir de 1990 : les Périmètres Irrigués Privés (PIP). Ces derniers sont élaborés sans mobilisation des ressources de l'État par des individus ou des groupes et sont destinés à la production vivrière (Seck, 2009). Leur essor est favorisé par la création de la Caisse Nationale de Crédit Agricole du Sénégal (CNCAS), l'assouplissement de la législation sur les coopératives en 1984, et l'édification du barrage anti-sel de Diama (1986) et celui de la 
régulation de Manantali (1988). Mais, c'est surtout le reversement, des terres classées en zone pionnière (décret 64-46 de 1964) aux zones de terroir en 1987, qui a encouragé les producteurs à financer et à exploiter en toute autonomie des périmètres hydroagricoles dans un contexte d'Ajustement Structurel. Entre 1989 et 2000, les PIP passent de 12500 ha à 33600 ha pour l'ensemble de la rive gauche et, dépassent 45500 ha en 2005 (Seck, 2009). Malgré ce développement rapide, les PIP vont montrer leurs limites, car les aménagements réalisés sont sommaires et de mauvaises qualités que beaucoup finiront par abandonner deux voire trois années plus tard (Bélières et Touré, 1999). Il y a une réelle absence de moyens financiers chez les populations locales (D’Aquino et al., 2017).

L'expérience des PIP aura tout de même montré une volonté des producteurs à investir dans l'agriculture irriguée. Ce facteur, combiné aux difficultés croissantes de l'État à mobiliser des financements extérieurs, a fait émerger au début des années 2000 l'idée de périmètres en Partenariat Public Privé (PPP). Lancés respectivement à partir de 2007 et 2009, le PDMAS et le 3PRD ont chacun aménagé 2500 ha. Le premier met l'accent sur le développement des cultures maraichères (oignon, tomate, piment, etc.) alors que le second s'intéresse à l'amélioration des performances de la riziculture irriguée. Le 3PRD a défini cinq catégories d'exploitations, allant de 5 ha à 50 ha tandis que le PDMAS a octroyé aux producteurs des parcelles allant de moins de 2 ha à 100 ha. Cette nouvelle forme d'attribution se démarque de celle de la SAED qui avait l'habitude d'attribuer environ 0,2 ha par actif dans les Grands Aménagements et les Aménagements Intermédiaires (Seck, 1981).

\section{L'accès à la terre}

\section{Des canaux d'accès à la terre très diversifiés}

Les ménages enquêtés possèdent au total une superficie de 2408 ha répartie en 549 parcelles, soit une moyenne de 7,4 ha/ménage (cf. tableau $\mathrm{n}^{\circ} 1$ ). Le chiffre élevé de l'écart type $(24,3)$ montre une forte distribution des valeurs autour de cette moyenne. Le minimum de superficie possédée par un ménage est de 0,10 ha et le maximum 350 ha, soit un écart de 340 ha, ce qui montre d'emblée l'inégalité de propriété foncière entre les producteurs. Le patrimoine foncier des ménages évolue souvent en fonction du rythme de la progression des aménagements hydroagricoles.

Tableau 1 : Présentation générale des parcelles recensées

\begin{tabular}{|c|c|c|c|c|}
\hline $\begin{array}{c}\text { Superficie } \\
\text { Moyenne par } \\
\text { Ménage (ha) }\end{array}$ & $\begin{array}{l}\text { Ecart } \\
\text { type }\end{array}$ & $\begin{array}{c}\text { Superficie } \\
\text { minimum } \\
\text { possédée par un } \\
\text { ménage (ha) }\end{array}$ & $\begin{array}{c}\text { Superficie } \\
\text { maximum } \\
\text { possédée par un } \\
\text { ménage (ha) }\end{array}$ & $\begin{array}{c}\begin{array}{c}\text { Nombre de } \\
\text { parcelles }\end{array} \\
\text { Parcelles } \\
\text { recensées }\end{array}$ \\
\hline 7,4 & 24,3 & 0,10 & 350 & 549 \\
\hline
\end{tabular}


Pour acquérir une superficie cultivable, les personnes enquêtées passent par plusieurs canaux (figure 3).

Figure 3. Canaux à travers lesquels les ménages obtiennent leurs parcelles

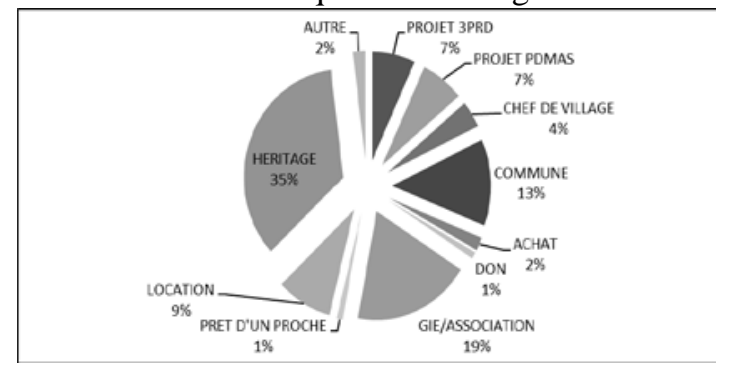

Source : auteurs

L'héritage demeure le premier mode d'accès à la terre avec $35 \%$ des réponses enregistrées. Cela montre que le droit foncier traditionnel reste une pratique très importante dans le Delta du fleuve Sénégal. L’héritage est suivi par les structures associatives (19\%), les affectations obtenues directement des communes (13\%), la location de terres (9\%). Il faut souligner que le PDMAS et le 3PRD participent ensemble à la disponibilité des exploitations agricoles à hauteur de $14 \%$. On note aussi qu'avec $4 \%$ des cas enregistrés, les chefs de villages gardent une certaine influence sur le foncier. Les autres canaux d'accès à la terre (le don, le prêt et l'achat) ne dépassent pas chacun les $2 \%$.

La figure 4 ci-dessous permet de comprendre les canaux d'accès dominants pour chaque catégorie de parcelles dans l'échantillon étudié.

Figure 4. Types de parcelles et canaux d'accès

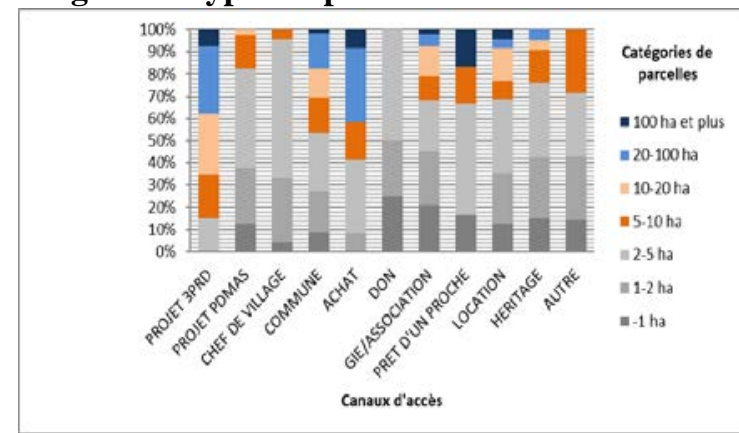

Source : auteurs

Les canaux d'accès aux "petites parcelles » sont dominés par l'héritage et les structures associatives. En ce qui concerne les "moyennes parcelles", c'est l'héritage qui prédomine également dans les modes d'accès. Toutefois, soulignons que l'accès grâce aux structures associatives est plus important que l'héritage dans la tranche des parcelles comprises entre 10-20 ha. On constate 
que les ménages enquêtés ont essentiellement bénéficié que de «petites parcelles » dans le projet PDMAS.

Pour les "grandes parcelles", les principaux canaux d'accès sont respectivement le projet $3 \mathrm{PRD}$, les affectations directes des communes, l'héritage, les structures associatives. Contrairement au projet PDMAS, les ménages enquêtés ont largement bénéficié de "grandes parcelles» dans le projet 3PRD. Mais, la plus importante part des ménagés bénéficiaires ont des superficies qui ne dépassent pas 100 ha.

\section{Une répartition inégale de la propriété foncière}

C’est la taille de l'ensemble des parcelles détenues par un ménage qui est considéré comme sa propriété foncière (George, 1963). Ainsi on distingue la petite propriété (moins de 5 ha) la moyenne propriété (entre 5 ha et 20 ha) et la grande propriété (plus de 20 ha). Souvent c'est en passant par divers canaux que les producteurs augmentent leurs patrimoines fonciers. Par exemple, ils peuvent hériter de leurs parents une parcelle, obtenir deux autres grâce à successivement leur GIE/associations et le 3PRD ou encore acheter de petites parcelles. Les combinaisons sont ainsi multiples.

Environ $72 \%$ des ménages enquêtés détiennent une petite propriété. L'essentiel de ces propriétaires dispose de parcelles dont les dimensions sont comprises entre $1-2$ ha (38\%) et entre $2-5$ ha (37\%), les très faibles surfaces de moins de 1 ha n'en représentent que $25 \%$ (figure 5). La moyenne propriété concerne $21 \%$ de nos enquêtés. Dans cette catégorie 64,5 \% de nos répondants possèdent des surfaces qui se situent entre 5-10 ha et 35,5\% détiennent un patrimoine foncier compris entre 10-20 ha.

Figure 5. Type de propriété foncière et pourcentage des ménages concernés

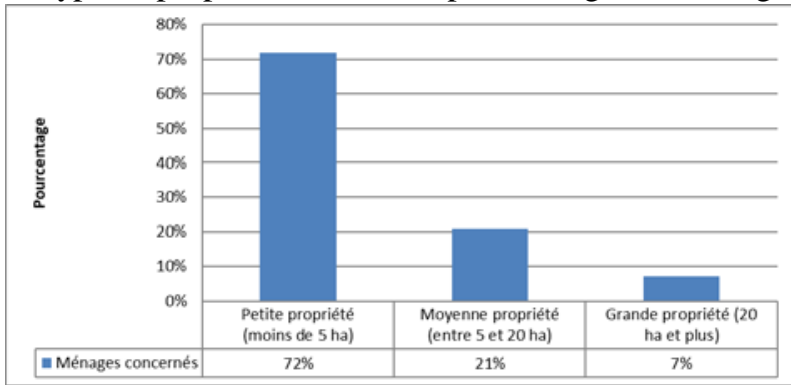

Source : auteurs

C'est seulement $7 \%$ des ménages enquêtés qui ont une grande propriété. Dans cette classe, $84 \%$ de nos interviewés ont entre 20-100 ha, et seulement $16 \%$ détiennent 100 ha et plus. Avec $30 \%$ des propriétaires de 100 ha et plus recensés, le 3PRD a permis le plus ces dernières années aux producteurs d'accroître leur patrimoine foncier. Dans les normes ce projet avait éliminé les parcelles de 100 ha qu'il devait attribuer. Toutefois certains 
bénéficiaires du 3PRD ont adopté des stratégies pour se retrouver avec ces superficies. Ils ont déposé pour deux parcelles de 50 ha en utilisant pour l'une leur nom et pour l'autre celui de leur fils, de leur épouse ou de leur entreprise.

\section{Mise en valeur des terres}

Grâce à la politique agricole de l'État tournée vers l'autosuffisance alimentaire et aux investissements consentis (Le Gal, 1989), la riziculture s'est imposée dans les terroirs étudiés. La maîtrise complète de l'eau dans le Delta du Sénégal offre aux ménages la possibilité de faire 3 campagnes agricoles : la contre-saison chaude (CSC), l'hivernage et la contre-saison froide (CSF) (tableau 2). Le riz est la principale spéculation exploitée en contre-saison chaude et en hivernage. Elle occupe $86 \%$ des parcelles exploitées en contresaison chaude et $97 \%$ des superficies emblavées. La production de riz baisse légèrement en hivernage tant en termes de parcelles exploitées (62 \%) qu'en superficie emblavée (92\%). Le système actuel de la production rizicole est alors beaucoup plus structuré autour la campagne de CSC qui se déroule entre janvier et juillet.

Par contre, la riziculture est faiblement pratiquée en contre-saison froide dans les communes de Diama et de Ross Béthio, comme partout ailleurs dans le Delta. Cette attitude s'explique par le fait que les producteurs n'obtiennent pas de bons rendements et les ressources limitées sont parfois orientées vers la production maraichère. Les rares paysans qui s'aventurent à la riziculture durant la contre-saison froide (septembre à mars) sont souvent ceux qui possèdent de petites superficies et préfèrent cultiver l'oignon et/ou d'autres produits horticoles durant la contre-saison chaude.

Tableau 2 : Occupation des terroirs par la riziculture durant les différentes saisons

\begin{tabular}{|c|c|c|c|c|}
\hline Variables & \multicolumn{2}{|c|}{ Superficie } & \multicolumn{2}{c|}{ Parcelle } \\
\hline Item & $\begin{array}{c}\text { Superficie } \\
\text { totale mise en } \\
\text { valeur (ha) }\end{array}$ & $\begin{array}{c}\text { Pourcentage } \\
\text { occupé par le riz } \\
(\%)\end{array}$ & $\begin{array}{c}\text { Nombre total } \\
\text { de parcelles } \\
\text { cultivées }\end{array}$ & $\begin{array}{c}\text { Pourcentage } \\
\text { occupé par le riz } \\
(\%)\end{array}$ \\
\hline $\begin{array}{c}\text { Contre-saison } \\
\text { chaude }\end{array}$ & 1631,63 & 97 & 368 & 86 \\
\hline Hivernage & 302,16 & 92 & 81 & 62 \\
\hline $\begin{array}{c}\text { Contre-saison } \\
\text { froide }\end{array}$ & 174,46 & 58 & 127 & 27 \\
\hline
\end{tabular}

Source : auteurs

Le faire-valoir direct représente $87 \%$ des modes de mise en valeur des parcelles recensées. C'est souvent la taille de la propriété foncière ainsi que les ressources humaines, matérielles et financières dont dispose le cultivateur, qui orientent le niveau d'utilisation de la main-d'œuvre familiale ou saisonnière. Mais dans l'ensemble des cas enquêtés, sur 475 parcelles en faire- 
valoir direct, 351 sont exploitées exclusivement par la famille, 122 par la famille et un/des sourga (s), seules 2 sont gérées par des sourgas uniquement. Le recours à une main d'œuvre permanente (sourga) dépend souvent de deux facteurs : une surface agricole plus grande que la capacité de mise en valeur de la famille ou l'éloignement de la parcelle par rapport au domicile du producteur. Ce dernier facteur peut même entrainer un faire-valoir indirect de la terre. Il a été très rare cependant de voir des cas de faire-valoir indirect dans l'effectif que nous avons enquêté, car il ne représente que $2 \%$ du mode de gestion des parcelles recensées.

Ainsi les terres possédées par les ménages enquêtés sont majoritairement mises en valeur. Sur les 549 parcelles recensées, seules, 63 sont inexploitées (11\%), quelle que soit la saison. Cette absence de mise en valeur est pratiquement proportionnelle à la distribution des types de parcelles. En effet, $66 \%$ des unités de production inexploitées sont de petite taille, $16 \%$ et $18 \%$ sont respectivement de moyenne et de grandes dimensions. On constate également que les canaux d'accès, tels que l'héritage, les communes et les structures associatives, qui offrent plus de possibilités d'accès à la terre aux petits producteurs, connaissent les niveaux de manque de mise en valeur les plus élevés avec respectivement $36 \%, 17 \%$ et $10 \%$ (figure 6 ). La figure 6 montre également que certaines parcelles issues du Partenariat Public-Privé restent encore en inexploitation.

Figure 6. Canaux d'accès aux terres inexploitées

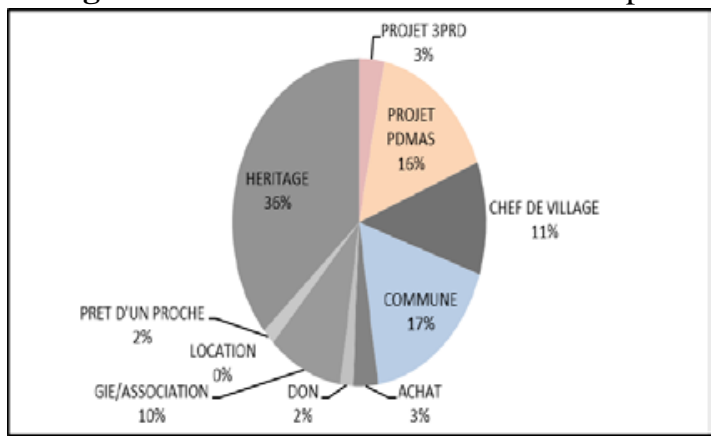

Source : auteurs

Environ $16 \%$ du total des terres inexploitées dans l'échantillon étudié ont été octroyés dans le cadre du PDMAS. D’ailleurs, $25 \%$ des parcelles de ce programme que nous avons recensées ne sont pas exploitées à ce jour. Les populations qui se sont regroupées en GIE (qui dépassent parfois 40 membres) et qui ont obtenu des parcelles situées entre 4 et 6 hectares peinent toujours à se les redistribuer et/ou à l'exploiter. Dans bien des cas, c'est le manque de moyens pour mobiliser les fonds nécessaires (3 500000 FCFA/ha) qui entraine le manque de mise en valeur de la terre. Actuellement ce sont les producteurs qui ont les possibilités d'aménager leurs périmètres, de financer 
leurs propres campagnes et de protéger leurs champs, qui exploitent les terres qu'ils ont obtenues dans le cadre du PDMAS. Pour le projet 3PRD, seules 2 parcelles n'étaient pas exploitées sur les 40 enquêtées.

\section{Analyse typologique : terres cultivées, revenus agricoles et pauvreté des ménages}

Peut-on établir un profil des ménages pauvres dans la zone étudiée à partir de leur patrimoine foncier, de leur niveau de vie et de leur activité agricole ? La tentative de trouver des réponses à cette interrogation nous a poussés à faire recours à une Classification Hiérarchique Ascendante (CAH). Le dendrogramme ci-dessous (figure 7) illustre le nombre de fusions terminales ainsi que le processus de regroupement qui résultent de cette $\mathrm{CAH}$. 5 groupes de ménages ont été créés selon la méthodologie décrite plus haut. Afin de faire l'étude typologique, une analyse bivariée (comparaison des moyennes) a été faite entre la nouvelle variable quantitative nominale découlant de la méthode de Ward et les 7 variables utilisées (Tableau 3).

Figure 7. Dendrogramme de la classification ascendante hiérarchique (CAH)

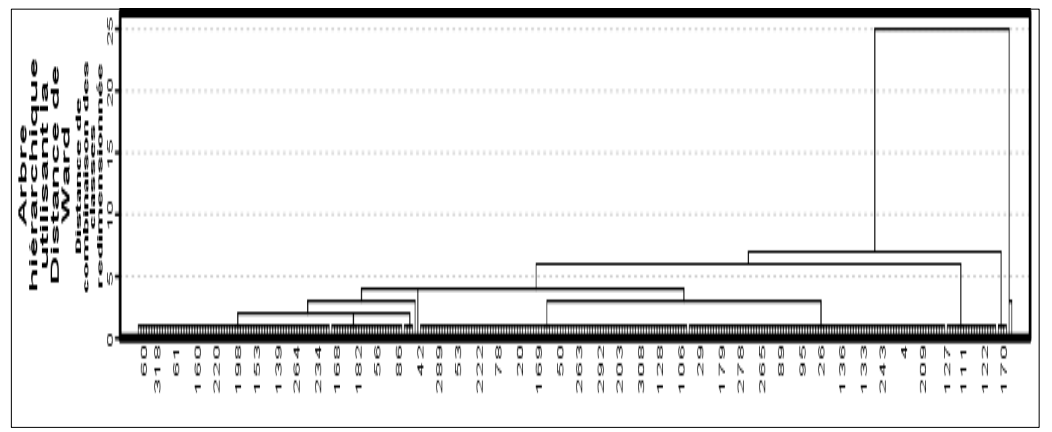

Les résultats globaux montrent que les ménages des agriculteurs enquêtés dans le Delta du fleuve Sénégal comptent en moyenne 12 membres. Ils cultivent 6 ha durant toutes les saisons possibles dans l'année, dont $83 \%$ ( 5 ha) en contre-saison chaude. Cette dernière fournit également $77 \%$ des bénéfices agricoles (marges) réalisés par les ménages soit la somme de 2593993 FCFA sur les 3368313 FCFA obtenus durant les trois saisons de cultures possibles. Ils dépensent en moyenne pour leur consommation quotidienne 152788 FCFA par mois et sur 100 ménages, 12 sont pauvres.

Cette analyse globale cache les disparités qui existent entre les 5 groupes ressortis de la classification hiérarchique ascendante. En considérant d'une manière croissante les ménages les plus pauvres jusqu'aux plus riches, le tableau 3 ci-dessous montre des différences remarquables.

- Le groupe 3 regroupe les ménages les plus pauvres (taux de pauvreté de $49 \%$ ) et 5,80 \% de l'effectif enquêté. Ils ne cultivent que 1 ha pendant les trois différentes saisons (généralement en contre-saison chaude) et gagnent 
440737 FCFA. Ils dépensent en consommation 102500 FCFA par mois alors que leur poids démographique est de 11 membres par ménage.

- Le groupe 1 représente plus de la moitié de l'échantillon (60,10\%). Il est caractérisé par des ménages présentant un taux de pauvreté de $12 \%$, qui ne cultivent que 2 ha durant les trois saisons (généralement en contre-saison chaude). Ils gagnent de l'agriculture 856114 FCFA, leurs ménages comptent 9 membres et font des dépenses de consommation mensuelles estimées à 116337 FCFA.

- Le groupe 2 (32,20 \% de l'effectif enquêté) isole ceux qui cultivent 8 ha lors des trois saisons et obtiennent du même coup une marge de 3480196 FCFA. Leur taux de pauvreté est moins élevé que les deux groupes précédents (6\%). Aussi, dépensent-ils plus que ces derniers (208 733 FCFA/mois) et ont également des ménages plus grands qu'eux (18 membres).

- Le groupe 4 concentre des ménages relativement aisés si on les juge par les superficies qu'ils emblavent pendant les trois saisons (92 ha en moyenne) et par les revenus qu'ils gagnent de l'agriculture (50 114750 FCFA). Leur taux de pauvreté est très faible (4\%) tout comme la part qu'ils occupent dans l'effectif enquêté (1,20 \%). Pourtant avec le même poids démographique que les ménages du groupe 2 (18 membres du ménage), ils ont toutefois un niveau de vie supérieur à ces derniers puisqu'ils dépensent mensuellement 312500 FCFA.

Tableau 2. 5 groupes de ménages classés selon 7 variables quantitatives

\begin{tabular}{|c|c|c|c|c|c|c|c|c|}
\hline \multicolumn{2}{|c|}{$\begin{array}{l}\text { Méthode de Ward } \\
\text { (groupes) }\end{array}$} & $\begin{array}{c}\text { Nombre } \\
\text { des } \\
\text { membres } \\
\text { du ménage }\end{array}$ & $\begin{array}{c}\text { Marge réalisée } \\
\text { toutes les trois } \\
\text { saisons } \\
\text { (FCFA) }\end{array}$ & $\begin{array}{c}\text { Marge réalisée } \\
\text { lors de la CSC } \\
\text { (FCFA) }\end{array}$ & $\begin{array}{c}\text { Superficie } \\
\text { cultivée lors } \\
\text { des trois } \\
\text { saisons (ha) }\end{array}$ & $\begin{array}{l}\text { Superficie } \\
\text { cultivée en } \\
\text { CSC (ha) }\end{array}$ & $\begin{array}{l}\text { Taux de } \\
\text { pauvreté }\end{array}$ & $\begin{array}{c}\text { Dépenses } \\
\text { moyennes } \\
\text { mensuelles } \\
\text { (FCFA) }\end{array}$ \\
\hline \multirow[t]{3}{*}{1} & Moyenne & 9 & 856114 & 607245 & 2 & 2 & $12 \%$ & 116337 \\
\hline & $\mathrm{N}$ & 196 & 196 & 196 & 196 & 196 & 196 & 196 \\
\hline & $\%$ de $\mathrm{N}$ total & $60,10 \%$ & $60,10 \%$ & $60,10 \%$ & $60,10 \%$ & $60,10 \%$ & $60,10 \%$ & $60,10 \%$ \\
\hline \multirow[t]{3}{*}{2} & Moyenne & 18 & 3480196 & 2780238 & 8 & 6 & $6 \%$ & 208733 \\
\hline & $\mathrm{N}$ & 105 & 105 & 105 & 105 & 105 & 105 & 105 \\
\hline & $\%$ de $\mathrm{N}$ total & $32,20 \%$ & $32,20 \%$ & $32,20 \%$ & $32,20 \%$ & $32,20 \%$ & $32,20 \%$ & $32,20 \%$ \\
\hline \multirow[t]{3}{*}{3} & Moyenne & 11 & 440737 & 339454 & 1 & 1 & $49 \%$ & 102105 \\
\hline & $\mathrm{N}$ & 19 & 19 & 19 & 19 & 19 & 19 & 19 \\
\hline & $\%$ de $\mathrm{N}$ total & $5,80 \%$ & $5,80 \%$ & $5,80 \%$ & $5,80 \%$ & $5,80 \%$ & $5,80 \%$ & $5,80 \%$ \\
\hline \multirow[t]{3}{*}{4} & Moyenne & 18 & 50114750 & 39406750 & 92 & 77 & $4 \%$ & 312500 \\
\hline & $\mathrm{N}$ & 4 & 4 & 4 & 4 & 4 & 4 & 4 \\
\hline & $\%$ de $\mathrm{N}$ total & $1,20 \%$ & $1,20 \%$ & $1,20 \%$ & $1,20 \%$ & $1,20 \%$ & $1,20 \%$ & $1,20 \%$ \\
\hline \multirow[t]{3}{*}{5} & Moyenne & 43 & 178010000 & 135310000 & 205 & 155 & $5 \%$ & 950000 \\
\hline & $\mathrm{N}$ & 2 & 2 & 2 & 2 & 2 & 2 & 2 \\
\hline & $\%$ de $\mathrm{N}$ total & $0,60 \%$ & $0,60 \%$ & $0,60 \%$ & $0,60 \%$ & $0,60 \%$ & $0,60 \%$ & $0,60 \%$ \\
\hline \multirow[t]{3}{*}{ Total } & Moyenne & 12 & 3368319 & 2593993 & 6 & 5 & $12 \%$ & 152788 \\
\hline & $\mathrm{N}$ & 326 & 326 & 326 & 326 & 326 & 326 & 326 \\
\hline & $\%$ de $\mathrm{N}$ total & $100 \%$ & $100 \%$ & $100 \%$ & $100 \%$ & $100 \%$ & $100 \%$ & $100 \%$ \\
\hline
\end{tabular}

Source : auteurs

Enfin le groupe 5 met en exergue deux ménages qui se détachent complètement du lot tant de par leur poids démographique (43 membres) que de par la superficie qu'ils réussissent à cultiver toutes les trois saisons 
possibles (205 ha). Ils gagnent ainsi 178010000 FCFA de l'agriculture (toute saison confondue) et dépensent en moyenne pour leur consommation $950000 \mathrm{FCFA} /$ mois. Ces ménages atypiques résident à Ross-Béthio mais sont originaires de Touba. Ils appartiennent à des dignitaires religieux qui doivent la taille de leur ménage au nombre de leurs disciples. Ils ont bénéficié, des parcelles dans le casier de Grande Digue Tellel, dans les PIP et dans le périmètre du 3PRD. Ils possèdent également des tracteurs ainsi que des moissonneuses batteuses en plus d'une main d'œuvre qui est à leur portée. Ce sont ces différents facteurs qui leur facilitent une riziculture intensive aussi intéressante que les bénéfices qu'ils réussissent à y réaliser. Ces résultats dénotent dans leur ensemble une forte relation entre le taux de pauvreté des ménages, leurs dépenses de consommation, leur poids démographique, la surface qu'ils cultivent durant les différentes saisons et les revenus agricoles qu'ils réussissent à glaner. Il est clair que ceux qui cultivent plus de terres obtiennent les meilleurs gains et affichent les dépenses de consommation les plus élevées. Les ménages les plus pauvres sont ceux qui emblavent moins de 2 ha durant toutes les campagnes, ils ont le niveau de vie ainsi que les revenus agricoles les plus bas.

\section{Discussion}

Il ressort de cette analyse une tendance à l'agrandissement de la taille des exploitations agricoles dans le Delta du Sénégal. Les ménages ne reçoivent plus les 0,20 ha par actif (Seck, 1981 ; Boutillier, 1982). Cet agrandissement qui a débuté depuis l'avènement des PIP est désormais accéléré par le Partenariat Public-Privé (surtout à travers le projet 3 PRD) et encadré actuellement par le Plan Sénégal Emergent. «En 1960, il y avait $58 \%$ d'exploitations agricoles sénégalaises de moins de 3 hectares sur $22 \%$ de la surface cultivée. En 1998, elles ne représentent plus que $51 \%$ du total sur seulement $16 \%$ de la surface cultivée. Au cours de la même période, la part des exploitations de plus de 10 hectares est passée de 6 à $9 \%$, et les surfaces qu'exploitent ces dernières sont plus importantes (de 26 à $33 \%$ du total)» (République du Sénégal, 2013).

Cette tendance sénégalaise d'élargissement des parcelles cultivées est l'inverse de ce qui se produit dans le monde et qui est cité comme étant l'un des facteurs de la crise alimentaire de 2008: la réduction des surfaces cultivables et des rendements sous l'effet de l'urbanisation et de l'augmentation de la population. Selon la CNUCED (2008) «à cause de la croissance démographique et de la perte de terres cultivables, la superficie moyenne des exploitations en Chine et au Bangladesh, par exemple, est tombée d'environ 1,5 hectare dans les années 70 à tout juste 0,5 hectare à l'heure actuelle; en Éthiopie et au Malawi, elle est tombée de 1,2 hectare à 0,8 hectare dans les années $90 »$. Cette tendance bien, qu'elle demeure 
favorable à l'augmentation des productions agricoles, accentue cependant les inégalités entre les ménages. Une minorité de producteurs se partage la grande part du patrimoine foncier alors que les ménages les plus pauvres se bousculent sur de petites superficies qu'ils sont obligés encore d'émietter, pour les héritiers, en cas de décès du chef de ménage.

En outre les modes d'accès aux parcelles agricoles sont différents, mais dans la majorité l'héritage, les communes, et les structures associatives sont les canaux qui permettent à la masse paysanne d'acquérir des unités de production. Ceux qui ont les moyens ou qui font partie des réseaux peuvent accroitre leurs superficies en achetant des terres et/ou en bénéficiant des aménagements effectués par les nouveaux projets (PDMAS, 3 PRD). C’est la conjugaison de l'ensemble de ces facteurs qui entraine une inégalité de la propriété foncière au sein des cultivateurs. L'écrasante majorité de ces derniers (63\%) exploite moins de 2 ha tandis que 7,5\% emblavent plus de 20 ha. Même si dans d'autres villages on peut rencontrer des cas isolés, la plupart des paysans qui ont une grande propriété habitent à Boundoum Barrage et surtout à Ross Béthio. Ce sont généralement des producteurs qui ont pratiquement des parcelles dans toutes les générations d'aménagements (Grand aménagement, PIP, 3PRD). Notre étude confirme ainsi les inégalités d'accès à la terre déjà évoquées par Le Roy (2006) dans le Delta du fleuve Sénégal. L'étude que ce chercheur a menée sur 429 exploitations dans 3 villages du Delta (Tiguette, Thiagar et Ndelle) montre 9 unités à la taille démesurée qui occupent $46 \%$ de la superficie cultivée tandis que, l'écrasante majorité, 362 exploitations ne s'étendent que de quelques ares à 12 hectares alors que 58 n'exerçaient aucune activité. La même situation a été constatée par Bélières et al. (2011) à l'Office du Niger où $74 \%$ des exploitations disposent seulement de $18 \%$ de la superficie, alors que $16 \%$ en cumulent $46 \%$.

Le résultat majeur qui ressort de cette étude est que les paysans du Delta du Sénégal qui ne cultivent pas assez de terres irriguées sont les plus pauvres. Il confirme à cet effet celui de Le Roy et al. (2005) qui, depuis plus d'une décennie, ont montré que certains ménages du Delta et de la Moyenne Vallée sont exclus de l'irrigation à cause de leur pauvreté, de leur endettement, de leur manque d'accès à la terre. Dans le cas des ménages que nous avons étudiés, le problème d'accès au crédit ne se pose pas, mais les producteurs ne cultivent pas assez de terre pour générer des revenus considérables. Cette problématique n'est pas singulière au Delta du fleuve Sénégal. En effet la course foncière dans l'Office du Niger qui favorise les plus riches au détriment des pauvres est aussi relatée par Adamczewski et al. (2013). Ailleurs, les recherches de Bainville et al. (2005) et d'Aubron, Lehoux et Lucas (2015) ont démontré que les ménages les plus pauvres respectivement au Nicaragua et en Inde rurale sont également ceux qui n'ont pas de terre ou pas d'eau pour les 
irriguer (manque d'un facteur de production). Au Brésil aussi, c'est la même situation qui est décrite par Di Méo (2014), les exploitations supérieures à 1000 ha constituent plus de $40 \%$ de l'espace agricole avec des propriétaires riches alors que les autres agriculteurs n'ont pas souvent accès au foncier et sont exploités par les grands propriétaires.

\section{Conclusion}

Dans le Delta du Sénégal, les inégalités sociales qui existent entre les ménages agricoles se manifestent à travers leur patrimoine foncier, leurs performances dans l'agriculture et leur niveau de vie. L'existence de plusieurs modes d'accès aux terres cultivables permet à une minorité de producteurs de détenir les plus grandes surfaces irrigables tandis que la majorité des ménages cultive moins de 2 ha. Un faible pourcentage des parcelles reste inexploité durant toute l'année montrant donc une certaine mise en valeur des terres. Mais malgré l'importance des terres exploitées, l'écrasante majorité des ménages, et surtout les plus pauvres, n’effectue qu'une seule campagne, la riziculture irriguée de contre-saison chaude.

En moyenne, les ménages les plus pauvres cultivent annuellement 1 ha et gagnent moins de $40000 \mathrm{FCFA} / \mathrm{mois}$. Les gains des ménages de classe moyenne se situent à $290016 \mathrm{FCFA} /$ mois pour 8 ha cultivés et ceux des ménages aisés avoisinent les 15 millions FCFA/mois pour 205 ha emblavées. Ce résultat montre une relation logique entre l'augmentation des superficies cultivées et des revenus agricoles. Le gouvernement du Sénégal est conscient de ce fait, c'est sans doute pour cette raison qu'il prône depuis la libéralisation l'agrandissement des unités de production agricole. C'est d'ailleurs dans cette même logique qu'il faudrait situer le 3PRD qui a attribué à ses bénéficiaires des parcelles supérieures ou égales à 5 ha selon le modèle du Partenariat Public Privé (PPP). Cependant, tel qu'il est actuellement conçu, le PPP fait que ce sont les riches cultivateurs qui risquent de profiter des nouvelles dotations foncières au détriment des plus pauvres. Une révision de la politique de développement de l'irrigation dans le Delta doit être guidée par la réponse à la question, comment les paysans les plus pauvres peuvent-ils s'affranchir de la pauvreté s'ils ne parviennent pas à cultiver plus de terre ?

\section{References:}

1. Adamczewski A., Jamin J.Y., Burnod P., Boutout Ly E. H., Tonneau J.P., 2013. «Terre, eau et capitaux : investissements ou accaparements fonciers à l'Office du Niger ?» Cah Agric 22: p. 22-32. doi : 10.1684/agr.2012.0601.

2. Aubron C., Lehoux H., Et Lucas C., 2015. « Pauvreté et inégalités en Inde rurale ", In: EchoGéo [En ligne], 32 |: http:// echogeo.revues.org/14226; DOI10.4000/echogeo.14226. 
3. Bainville S., Mena R., Rasse-Mercat E., Touzard I., 2005. «La pauvreté des exploitations familiales nicaraguayennes: retard technique ou manque de terre ?», In : Revue Tiers Monde, $3 \mathrm{n}^{\circ} 183$, p. 559-580, https://www.cairn.info/revue-tiers-monde-2005-3-page559.htm.

4. Bélière J-F Et Touré E.A, 1999. Impact de l'ajustement structurel sur l'agriculture irriguée du delta du Sénégal, thèse de doctorat de l'Ecole Nationale Supérieure Agronomique de Montpellier, 491 p. et annexes.

5. Belieres J.F., Hilhorst T., Kebe D., Keïta M.S., Keïta S., Et Sanogo O., 2011. "Irrigation et pauvreté : le cas de l’Office du Niger au Mali », In: Cah. Agric. 20, p. 144-149. doi : 10.1684/agr.2011.0473.

6. Bourgoin J., Valette E., Diop D., Adamczewski A., Dia D., Toure L., 2016. «Le delta du fleuve Sénégal: quel modèle agricole?» In: Pesche D., Losch B., Imbernon J., (dir.). Une nouvelle ruralité émergente, Regards croisés sur les transformations rurales africaines, Atlas pour le Programme Rural Futures du Nepad, deuxième édition revue et augmentée, Montpellier, Cirad, Nepad, p. 52-53.

7. Boutillier J-L., 1982. «L'aménagement du fleuve Sénégal et ses implications foncières », In : LE BRIS E., Le ROY E., et Leimdorfer F., (dir.), Enjeux foncier en Afrique noire, Paris, Karthala, p. 301-308.

8. Boutillier J. L., 1989. "Irrigation et problématique foncière dans la vallée du Sénégal», In : cah. sci.hum 25 (4), Paris, Orstom, p. 469488.

9. CNUCED, 2008. Répondre à la crise alimentaire globale. Les politiques essentielles pour le commerce, l'investissement et les produits de base afin d'assurer la sécurité alimentaire durable et d'atténuer la pauvreté, ONU, New York et Genève, 60 p.

10. D’aquino P., Camara S. Et Seck S.M., 2017. «Formaliser ou sécuriser les droits locaux sur la terre ?», In: Études rurales URL: http://journals.openedition.org/etudesrurales/11590;DOI :10.4000/et udesrurales.11590.

11. Di Méo G., 2014. «Richesse et pauvreté : esquisse d’une géographie sociale du monde », In : Charvet J.P, Sivignon M. (dir.), Géographie humaine. Questions et enjeux du monde contemporain, p. 115-142.

12. Drieu O., Ndiaye O. K, Mbodj I., 2011. Etudes Aps, Apd Et Dee du schéma d'aménagement des réseaux secondaires d'alimentation et de drainage ainsi que des pistes de desserte de 2500 ha d'irrigation privée dans la zone du Gorom Aval; Etude d'Impact Environnemental et Social, $246 \mathrm{p}$.

13. IPA, 2018. Sénégal, PPI 2011 guide d'utilisation, 30 p.

14. Le Roy X., Kane M., Marcellin C., Sy M., Ball O., 2005. « Pauvreté et accès à l'eau dans la vallée du Sénégal », In: Pauvreté Hydraulique et 
Crises Sociales: Colloque International, Décembre, Agadir, Maroc,16p.

15. Le Roy X., 2006. «Agriculture irriguée et inégalités sociales dans la vallée du fleuve Sénégal », PCSI - 4e Séminaire international et interdisciplinaire, Montpellier, France, CIRAD, 13 p. <cirad00153767>.

16. Lerricolais A., Et Seck S.M., 2002. " Patrimoines anciens et territoires en gestation dans la région riveraine du fleuve Sénégal », In: Patrimonialiser la nature tropicale: Dynamiques locales, enjeux internationaux, Paris, IRD Editions, p. 283-300.

17. Maertens M., Colen L., Et Swinnen J.F.M., 2011. « Globalisation and poverty in Senegal: a worst case scenario?” In: European Review of agricultural Economics, Vol 38 (1), p. 31-54.

18. République Du Sénégal, 2013. Plan Sénégal Émergent, 107 p.

19. Saed, 2009. Rapport annuel d'activités 2008, PDMAS, 16 p.

20. Seck S.M., 1981. Irrigation et aménagement de l'espace dans la moyenne vallée du Sénégal : participation paysanne et problème de développement, thèse de doctorat d'Etat, Université de Saint-Etienne, $630 \mathrm{p}$.

21. Seck S.M, 2009. "Changements institutionnels et difficultés de développement hydroagricole dans le delta du fleuve Sénégal: nouvelles dynamiques et recomposition autour de l'irrigation", In : Dansero E., Luzzati E., Seck S.M., (dir.) : organisation paysanne et développement local : leçons à partir du cas du Delta du fleuve Sénégal, l’Harmattan, Italia, p. 21-52.

22. Seck S.M., 1991. "La dynamique de l'irrigation dans la vallée du fleuve », In : Crousse B., Mathieu P., Seck S.M., (sous la dir.), la vallée du fleuve Sénégal, évaluations et perspectives d'une décennie d'aménagements, Paris, Karthala, p. 17-44.

23. Soullier G., Moustier P., Bourgoin J. Et Ba A., 2019. «Les effets des investissements d'agrobusiness sur les agriculteurs familiaux. Le cas de la vallée du fleuve Sénégal ", In: Économie rurale, URL : http://journals.openedition.org/economierurale/6262 ;DOI :10.4000/e conomierurale.6262.

24. Sy K., 2014. Industrie et développement territorial : l'insertion des sociétés agro-industrielles dans le Delta et la basse vallée du fleuve Sénégal (rive gauche), thèse de doctorat de géographie de l'Université Paris Ouest de France et Gaston Berger du Sénégal, 313 p. 